\title{
Chemical Exchange Saturation Transfer (CEST) Imaging: Description of Technique and Potential Clinical Applications
}

\author{
Feliks Kogan · Hari Hariharan · Ravinder Reddy
}

Published online: 14 February 2013

(c) Springer Science+Business Media New York 2013

\begin{abstract}
Chemical exchange saturation transfer (CEST) is a magnetic resonance imaging (MRI) contrast enhancement technique that enables indirect detection of metabolites with exchangeable protons. Endogenous metabolites with exchangeable protons including many endogenous proteins with amide protons, glycosaminoglycans, glycogen, myoinositol, glutamate, creatine and several others have been identified as potential in vivo endogenous CEST agents. These endogenous CEST agents can be exploited as non-invasive and nonionizing biomarkers of disease diagnosis and treatment monitoring. This review focuses on the recent technical developments in endogenous in vivo CEST MRI from various metabolites as well as their potential clinical applications. The basic underlying principles of CEST, its potential limitations and new techniques to mitigate them are discussed.
\end{abstract}

Keywords MRI - CEST - Chemical exchange ·

Molecular imaging $\cdot$ GluCEST $\cdot$ APT

\section{Introduction}

Many methods have been proposed for noninvasive molecular imaging in vivo, but few so far have widespread

\author{
F. Kogan · H. Hariharan · R. Reddy $(\square)$ \\ Department of Radiology, Center for Magnetic Resonance \\ and Optical Imaging (CMROI), University of Pennsylvania, \\ B1 Stellar-Chance Laboratories, 422 Curie Boulevard, \\ Philadelphia, PA 19104-6100, USA \\ e-mail: krr@mail.med.upenn.edu \\ F. Kogan \\ e-mail: fkogan@seas.upenn.edu \\ H. Hariharan \\ e-mail: harih@mail.med.upenn.edu
}

applications in the clinical setting. While nuclear medicine techniques such as positron emission tomography (PET) have exquisite sensitivity, their primary shortcomings are the use of radioactive isotopes, many with short half-lives, and suboptimal spatial resolution [1]. Optical imaging on the other hand has a limited set of clinical applications due to poor depth of penetration [2]. Magnetic resonance spectroscopy (MRS) has been used for in vivo quantifications of concentrations of a wide range of metabolites in vivo and has shown that the concentrations of many of these metabolites are high enough for detection with MR [3-5]. However, poor spatial resolution and long acquisition times make the use of current MRS challenging for clinical use. Magnetic resonance imaging (MRI) is a nonionizing, high-resolution imaging technique, which is widely utilized clinically. However, current MRI-based molecular imaging techniques have relied primarily on exogenous contrast agents, which affect bulk water relaxation properties for contrast $[6,7]$. There is thus a need to develop noninvasive, high-resolution and quantitative methods to measure molecular changes in vivo.

Chemical exchange processes and their effects on the nuclear magnetic resonance (NMR) spectrum were some of the main topics of investigation that led to several key advancements in the early days of NMR [8-10]. However, only recently have these processes been exploited for contrast on MRI through saturation transfer experiments [11].

Chemical exchange saturation transfer (CEST) is a new contrast enhancement technique that enables the indirect detection of molecules with exchangeable protons and exchange-related properties [12, 13]. CEST makes MRI sensitive to the concentrations of endogenous metabolites and their environments.

CEST agents, molecules with exchangeable protons, can be divided into two classes: paramagnetic CEST agents 


\section{Solute}

Pool $\left(P_{s}\right)$

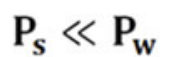

\section{Water}

Pool $\left(P_{w}\right)$

(a)

(b)<smiles>O=C(O)CCNNC(CCC(=O)O)C(=O)O</smiles>
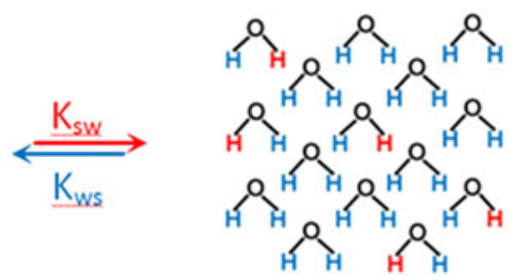

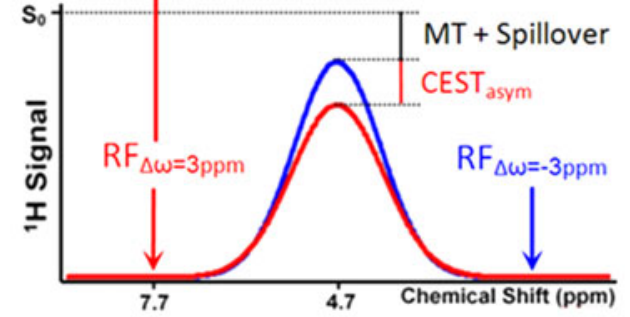

(c)

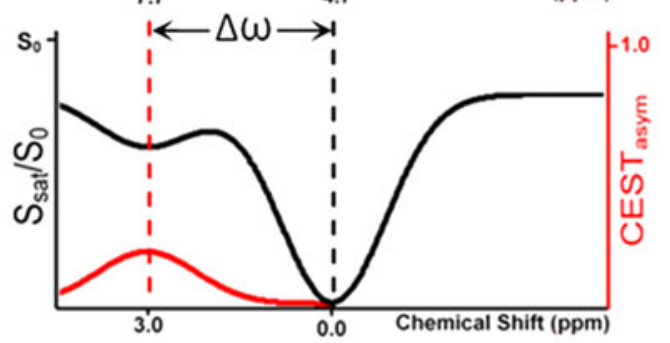

(d)

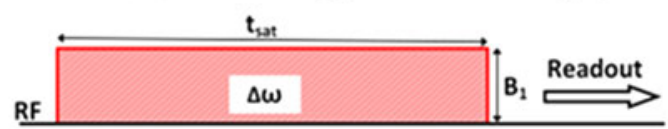

Fig. 1 Chemical exchange saturation transfer (CEST) contrast enhancement mechanism illustrated with a two-site exchange between a solute pool and a solvent pool (water). a Radiofrequency (RF) saturation applied at the resonance frequency $(\Delta \omega)$ of the labile solute protons $\left(P_{\mathrm{s}}\right)$ leads to a loss of net magnetization. These saturated protons (red) from the solute pool then exchange with unsaturated protons (blue) from the much larger water pool $\left(P_{\mathrm{w}}\right)$ with an exchange rate, $k_{\mathrm{sw}}$ leading to an accumulation of saturated protons in the water pool. b The accumulation of the zero net magnetization of solute protons in water results in a decrease in the total water signal. While the saturation pulse is being applied, this process continues to decrease the water magnetization through the CEST effect as well as through magnetization transfer (MT) and direct water saturation or "spillover" effects. A saturation pulse applied at the corresponding reference frequency symmetrically at the opposite side of the water resonance $(-\Delta \omega)$ will decrease the water magnetization through MT and spillover effects only. c Saturation transfer effects can be assessed using a $\mathrm{z}$-spectrum (black curve) where the water signal is plotted as a function of saturation frequency. Here the water resonance frequency is used as the center frequency and assigned the chemical shift of $0 \mathrm{ppm}$ as opposed to in NMR spectra, where water protons have a chemical shift of $4.7 \mathrm{ppm}$. Asymmetry analysis $\left(\mathrm{CEST}_{\text {asym }}\right)$ is performed by subtracting the water signal from one side of the $\mathrm{z}$-spectrum from the other side to mitigate the effects of spillover as well MT effects and isolate the effects of chemical exchange. d Standard CEST magnetization preparation consisting of a long saturation pulse applied at a resonance frequency, $\Delta \omega$, at a saturation amplitude, $B_{1}$, and duration $t_{\text {sat }}$. The saturation pulse can be a single, long, frequency-selective rectangular pulse, as shown here, or a train of shaped frequency-selective pulses separated by small delays

(PARACEST) and diamagnetic CEST agents. Molecules with exchangeable protons capable of providing CEST contrast combined with a paramagnetic metal ion (typically one of the lanthanides) are known as PARACEST agents. On the other hand, diamagnetic CEST agents are simply molecules with exchangeable protons without paramagnetic ions. PARACEST agents create larger chemical shifts between exchangeable protons, which allow for more selective irradiation and imaging of faster exchanging species [14-16]. While these PARACEST agents have promising applications, a detailed discussion of these is beyond the scope of this review. The reader is referred to several excellent reviews summarizing the PARACEST literature [17-19].

This review focuses on the recent developments in endogenous diamagnetic CEST methods and their potential clinical applications. Briefly, we outline the theoretical aspects of CEST and review recent developments in endogenous CEST contrast from amides on proteins, amine groups on small metabolites such as glutamate (Glu), creatine $(\mathrm{Cr})$ and hydroxyl groups on glycosaminoglycans (GAG), glycogen, myoinositol (MI), and glucose. Finally, the basic underlying principles and some of the limitations of CEST imaging as well as new methods to alleviate them are outlined. For a thorough discussion of theoretical aspects of CEST, the reader is referred to several outstanding reviews [19-21] on this topic.

\section{Theory}

Let us consider a two-site exchange process involving a solute pool $\left(P_{\mathrm{s}}\right)$ with exchangeable protons and a much larger solvent (water) pool $\left(P_{\mathrm{w}}\right)$. In CEST imaging, a frequency-selective radiofrequency (RF) saturation pulse is applied to the solute pool (Fig. 1d). A long saturation pulse, applied at the resonance frequency of the solute protons, equalizes the number of spins aligned against the magnetic field to those aligned with the magnetic field, leading to no net magnetization and resulting in the process termed "saturation", the net result of which is zero MR signal. This zero magnetization of saturated protons from the solute pool then exchanges with unsaturated protons from the much larger water pool, leading to decrease in the water signal proportional to the concentration of solute (Fig. 1a). While the saturation pulse is being applied, this process continues to decrease the water magnetization. Concurrently, longitudinal relaxation processes return the saturated proton spins to their thermal equilibrium state until the system reaches steady state or the saturation pulse is turned off. The reduction in the water signal can be imaged with any routine imaging sequences.

CEST contrast requires that a discrete chemical shift difference $(\Delta \omega)$ between water and the exchangeable 
proton on the solute is preserved, and the exchange rate, $k_{\mathrm{sw}}$, has to fulfill the slow to intermediate exchange condition on the NMR time scale defined as [22].

$k_{\mathrm{sw}} \leq \Delta \omega$

Generally, the saturation pulses are not perfectly frequency-selective and therefore lead to some direct saturation of the water protons, or "spillover" effects (Fig. 1b). Additionally, in biological tissues, the saturation of solute pools also causes magnetization transfer (MT) between water molecules bound to larger macromolecules in solid or semisolid phases and free water protons, which also leads to a decrease in the water signal. These different saturation transfer effects can be assessed using a $\mathrm{z}$-spectrum generated by plotting the water signal as a function of saturation frequency. Since the direct water saturation effects are symmetric with respect to the water resonance frequency, they can be removed by asymmetry analysis where the water signal from one side of the z-spectrum is subtracted from the other side [20] (Fig. 1c). Under certain saturation parameters, asymmetry analysis will also remove the contribution of MT. Thus, to isolate the chemical exchange effects of a particular metabolite, the CEST asymmetry ratio ( $\mathrm{CEST}_{\text {asym }}$ ) is computed by subtracting the normalized magnetization signal at the exchangeable solute proton frequency $\left[M_{\mathrm{sat}}(+\Delta \omega)\right]$ where $\Delta \omega$ is the chemical shift difference between solute and labile protons, from magnetization at the corresponding reference frequency symmetrically at the opposite side of the water resonance $\left[M_{\mathrm{sat}}(-\Delta \omega)\right]$ :

$\mathrm{CEST}_{\text {asym }}=\frac{M_{\text {sat }}(-\Delta \omega)-M_{\text {sat }}(+\Delta \omega)}{M_{\mathrm{ctl}}}$

where $M_{\mathrm{ctl}}$ is the control magnetization. For $M_{\mathrm{ctl}}$, either $M_{0}$, the magnetization observed with no saturation, the magnetization observed with a saturation pulse far from the water resonance $(\geq 20 \mathrm{ppm})$, or the $M_{\text {sat }}(-\Delta \omega)$ magnetization can be used [23].
In interpreting the CEST effect, factors that play a role are the concentration of the solute, the proton exchange rate, the number of exchangeable protons, the $\mathrm{pH}$ of the local environment, $T_{1}, T_{2}$, the saturation efficiency, and the amplitude and duration of the saturation pulse. These effects can be incorporated into a general solution obtainable from the analysis of a two-site exchange model in the presence of RF saturation [24, 25].

As $\Delta \omega$ increases linearly with static field strength, CEST imaging greatly benefits from ultra-high magnetic fields. As a result, molecules with high exchange rates, which do not satisfy the condition in Eq. (1) at lower fields $(\leq 3 \mathrm{~T})$, may still demonstrate a CEST effect at 7T.

While the chemical shift difference is directly related to the magnetic field strength, the chemical exchange rate depends mainly on the exchange type and environment. In vivo, the exchange rate is highly sensitive to changes in tissue $\mathrm{pH}$ [26]. The chemical exchange rate can change by several orders of magnitude with changes in $\mathrm{pH}$ as small as $1 \mathrm{U}$. It is therefore critical to identify endogenous agents whose chemical exchange rates satisfy Eq. (1) under physiological conditions. This exquisite sensitivity of exchange rate to $\mathrm{pH}$ can be exploited to measure $\mathrm{pH}$ using CEST-based MRI methods.

\section{Endogenous CEST}

Many of the metabolites originally examined for use as exogenous contrast agents are found endogenously at concentrations high enough for detection [22]. The feasibility of endogenous CEST imaging was first demonstrated in imaging of urea in the bladder of healthy human subjects [13]. Since then, several endogenous metabolites with exchangeable protons [amide $(-\mathrm{NH})$, amine $\left(-\mathrm{NH}_{2}\right)$ and hydroxyl $(-\mathrm{OH})$ groups] with optimal exchange properties under physiological conditions have been identified and imaged in vivo (see Table 1). Thus, CEST-based MRI

Table 1 Approximate CEST exchange properties for amide $(-\mathrm{NH})$, amine $\left(-\mathrm{NH}_{2}\right)$, and hydroxyl $(-\mathrm{OH})$ groups

\begin{tabular}{|c|c|c|c|}
\hline & $\begin{array}{l}\text { Amide protons } \\
(-\mathrm{NH})\end{array}$ & Amine protons $\left(-\mathrm{NH}_{2}\right)$ & Hydroxyl protons $(-\mathrm{OH})$ \\
\hline Chemical shift & $3.5 \mathrm{ppm}$ & $1.8-3.0 \mathrm{ppm}$ & $0.5-1.5 \mathrm{ppm}$ \\
\hline $\begin{array}{l}\text { Exchange rate }\left(k_{\mathrm{sw}}\right) \\
\text { range }\end{array}$ & $10-100 \mathrm{~s}^{-1}$ & $>500 \mathrm{~s}^{-1}$ & $500-1,500 \mathrm{~s}^{-1}$ \\
\hline Endogenous metabolites & Multiple unknown & Glutamate $(\mathrm{Glu})$, creatine $(\mathrm{Cr})$ & $\begin{array}{l}\text { Glycosaminoglycans (GAG), glycogen, } \\
\text { myoinositol (MI), glucose }\end{array}$ \\
\hline $\mathrm{CEST}_{\mathrm{asym}}^{\mathrm{a}}$ & $1-4 \%$ & $7-10 \%$ & $2-8 \%$ \\
\hline Sensitivity to $\mathrm{pH}$ & Yes & Yes & Yes \\
\hline CEST applications & Cancer/stroke & $\begin{array}{l}\text { Skeletal muscle and myocardial muscle } \\
\text { energetics, cancer metabolism }(\mathrm{Cr}) \text {, } \\
\text { neuropsychiatric disorders }(\mathrm{Glu})\end{array}$ & $\begin{array}{l}\text { Osteoarthritis (GAG), neurological disorders } \\
\text { (MI), cancer metabolism (glucose) }\end{array}$ \\
\hline
\end{tabular}

${ }^{a}$ At $7 \mathrm{~T}$ under physiological conditions 
methods show promise to use MRI as a noninvasive, nonionizing tool for molecular imaging.

\section{Amide Proton (-NH) Transfer (APT)}

The CEST effects from amide protons were first demonstrated in the rat brain at $4.7 \mathrm{~T}$, and this method was referred to as amide proton transfer (APT) [27]. Amide protons have a chemical shift $3.5 \mathrm{ppm}$ down field from water, which corresponds to the amide resonance at about $8.3 \mathrm{ppm}$ in the NMR spectrum [28]. Additionally, due to their very slow exchange rate $\left(\sim 30 \mathrm{~s}^{-1}\right)$ [29], it is possible to obtain almost complete saturation using a low power, long duration saturation pulse. However, when using this saturation scheme, there are MT effects which are not symmetric around the water resonance and thus create asymmetry of their own [30]. Nonetheless, when investigating diseased tissue such as tumors or ischemia, changes in $\mathrm{pH}$ affecting the amide proton exchange rate as well as changes to water content and the $R_{1}$ of water are able to provide contrast.

APT imaging has been performed for a range of applications. The first in vivo studies focused on characterizing changes in APT asymmetry from ischemic tissue induced by stroke in rats [27]. A 1-3\% decrease in APT asymmetry was seen following occlusion of the middle cerebral artery (MCA) assessed by APT asymmetry curves. This decrease was attributed to a decrease in proton exchange rate due to the decrease in $\mathrm{pH}$ resulting from ischemia. This was followed by APT imaging of tumors in cancer models [28]. In 9L gliosarcoma tumor rat models, an increase of 3-4\% was observed in the tumor compared to normal brain tissue in the contralateral hemisphere. This increase was hypothesized to be due to increased amide proton content in the brain tumors.

Recently, imaging of stroke and tumors has been applied to human models [31]. APT imaging was performed in human brain tumor patients showing an increase in APT in tumor regions. Furthermore, APT could differentiate the brain tumor regions from peritumoral edema and normal appearing white matter. More recent studies have demonstrated the feasibility of APT imaging for tumor grading $[32,33]$, which was further extended to studies of radiation necrosis. Fluid-attenuated inversion recovery (FLAIR) and gadolinium $(\mathrm{Gd})$-enhanced $T_{1}$-weighted images are routinely used to determine the extent of tumor involvement and to assess the therapeutic response. However, these methods cannot reliably distinguish between tumor recurrence and

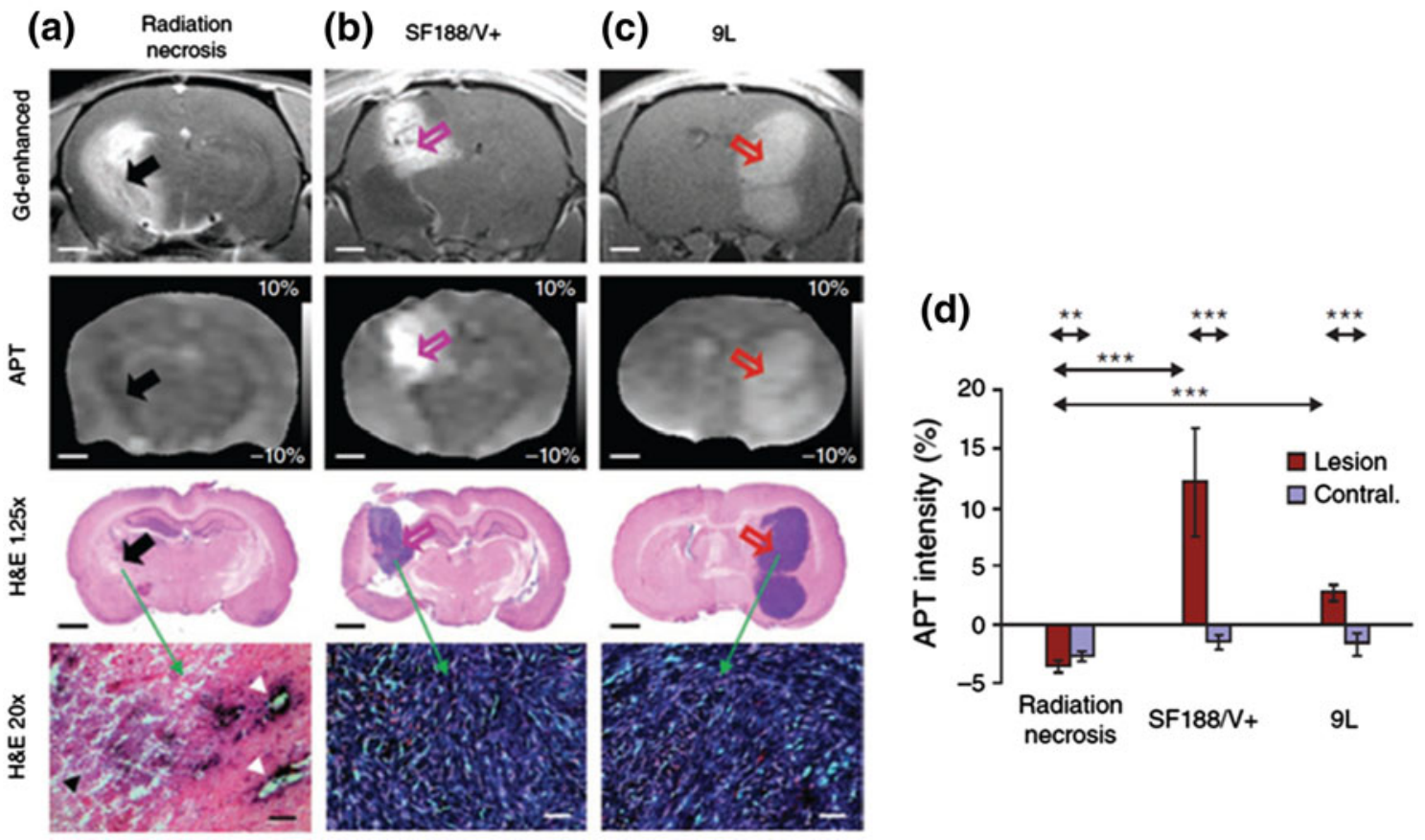

Fig. 2 Comparison of active tumors and radiation necrosis using APT MRI and histology. a Gadolinium (Gd) enhanced and APT MRI and $\mathrm{H} \&$ E-stained histopathological sections of a radiation necrosis (black arrowhead), b SF188/V+ human glioma tumor (pink open arrow), and c 9L gliosarcoma tumor (red open arrow). All three lesions appear hyperintense compared to contralateral brain tissue in Gd-enhanced MR imaging. However, on APT maps, active tumors appear hyperintense while radiation necrosis is hypointense to isointense. This corresponds to the high cellularity seen in histology of active tumors compared to radiation necrosis. d Quantitative comparison of APT image intensities (in percentage change of bulk water signal intensity) for radiation necrosis and active gliomas. Radiation necrosis and active tumors have opposite APT signal intensities with respect to the control contralateral brain tissue (from Ref. [34•], with permission.) 
radiation necrosis. APT could differentiate between active orthotopic gliomas that appear hyperintense from radiation necrosis, which appears hypointense (Fig. 2) [34•]. In addition, the APT intensity was shown to decrease in irradiated tumors from baseline to 3 and 6 days post treatment. New applications of APT imaging are emerging in studies of multiple sclerosis [35], breast cancer [36], prostate and bladder cancer [37], spinal cord imaging [38] as well as others. These applications could further increase the utility of CEST as a biomarker of disease.

Given the slow exchanges rate of amide protons, the APT experiments can be performed at $3 \mathrm{~T}$ as well as at higher fields. APT imaging shows promise in detecting and characterizing changes in various types of tumors, stroke, multiple sclerosis and other pathologies [21]. However, at present, it is not clear whether it can separate $\mathrm{pH}$ changes from protein content changes. Also, APT measurement is affected by MT asymmetry and nuclear overhauser effects (NOE). Therefore, to realize the full potential of APT, methods need to be developed to remove confounding effects such as MT asymmetry and NOE. Nonetheless, the slow exchange rate and relatively high concentrations of amide protons create conditions, which potentially allow this technique to be translated to clinical applications as an "index" of molecular changes.

\section{Hydroxyl (-OH) Protons}

Another important application of CEST imaging is in studying exchange of $-\mathrm{OH}$ groups in metabolites such as, glycogen, GAG, MI, and glucose. Recently, the feasibility of detecting the CEST effect from -OH groups of glycogen (glycoCEST) [39•] was demonstrated in vitro as well as in vivo in animal studies of the liver at 4.7T. GlycoCEST may have clinical utility in disorders of glycogen metabolism as well as conditions such as obesity, insulin resistance, and type 2 diabetes, where glycogen content may be abnormal.

Another important application of hydroxyl proton CEST imaging is GAG imaging in cartilage (GagCEST) [40]. GAGs are side chains attached to core proteins of proteoglycans. Loss of proteoglycans in cartilage tissue is the predominant initiating event in osteoarthritis [41]. Thus, GAG quantification may potentially be a biomarker for osteoarthritis. While a $20 \%$ GagCEST asymmetry was originally reported in cartilage at 3T, later studies showed that proper correction of $B_{0}$ inhomogeneities lead to negligible GagCEST contrast at 3T (Fig. 3a-c) [42•]. This is mainly due to direct saturation effects from the saturation scheme necessary to saturate faster exchanging spins with a small chemical shift. Nevertheless, moving to $7 \mathrm{~T}$, the chemical shift is increased leading to lower direct water saturation and makes GagCEST mapping possible in the knee. At $7 \mathrm{~T}$, a $\sim 6 \%$ GagCEST asymmetry was shown from patellar cartilage of human knees with optimized pulse parameters and correction for $B_{0}$ and $B_{1}$ field inhomogeneities. Studies at $7 \mathrm{~T}$ have shown a high correlation between GagCEST and sodium $\left({ }^{23} \mathrm{Na}\right)$ imaging in the patellar cartilage (Fig. 3d-f) [43]. Sodium imaging uses positively charged ${ }^{23} \mathrm{Na}$ in cartilage to map the concentration of negatively charged GAGs. Thus its high correlation with sodium imaging implies that GagCEST is potentially a useful biomarker for GAG. In addition, the inherent higher spatial resolution of the GagCEST technique as compared to sodium MRI is beneficial in imaging thin cartilage. Similar studies performed ex vivo on intervertebral discs demonstrate the feasibility of applying this technique for early diagnosis and characterization of disc degeneration and possibly for other orthopedic applications [44-46].

MI is another CEST agent with exchangeable hydroxyl protons that has been discussed for endogenous CEST. MI is a sugar-like molecule, with six $-\mathrm{OH}$ groups, located mainly in glial cells, which functions as an osmolyte, and is one of the most abundant metabolites visible in brain MR spectroscopy. Its concentration is altered in many brain disorders. The MI hydroxyl proton exchange rate is in the slow to intermediate exchange regime $\left(\sim 600 \mathrm{~s}^{-1}\right)$ and exhibits a concentration dependent CEST asymmetry which is maximized at $\sim 0.6 \mathrm{ppm}$ [47•]. In vivo studies demonstrated the feasibilities of MICEST Brain mapping. A significantly higher MICEST asymmetry was observed in 5 subjects from white matter $(5.2 \pm 0.5 \%)$ compared to gray matter $(4.3 \pm 0.5 \%)$. More recently, MICEST has been applied to a transgenic mouse model of Alzheimer's disease (AD) [48]. $\mathrm{AD}$ mice showed a $50 \%$ higher MICEST asymmetry than age matched wild type controls (Fig. 4). This was validated with increased MI concentration in MR spectroscopy as well as immunohistochemistry, which showed a proliferation of glial cells. As a glial marker, CEST imaging of MI could be a biomarker for a variety of neurological disorders.

Another important metabolite with exchangeable $-\mathrm{OH}$ proton groups is glucose. Recently, $-\mathrm{OH}$ groups of glucose have been exploited in imaging glucose in phantoms as well as in in vivo systems (GlucoCEST) [39•, 49-51]. Tumors typically rely more on glycolytic metabolism than normal tissues due to hypoxia or inhibited mitochondrial function and as a result, upregulated glucose metabolism is commonly used to detect and characterize tumors with ${ }^{18} \mathrm{~F}$ labeled fluorodeoxyglucose (FDG) PET. Similarly, preferential uptake of injected D-glucose in tumors can be imaged with GlucoCEST. Significant GlucoCEST signal enhancement has been shown at $11.7 \mathrm{~T}$ in mice in two human breast cancer cell lines during systemic sugar infusion [51]. These results show the potential of cancer detection and characterization with MRI using the GlucoCEST effect from simple non-toxic sugars. 
Fig. $3 B_{0}$ and $B_{1}$ corrected GagCEST maps of human knee cartilage at $\mathbf{a} 3 \mathrm{~T}$ and $\mathbf{b} 7 \mathrm{~T}$. c GagCEST asymmetry plot simulations at $3 \mathrm{~T}$ and $7 \mathrm{~T}$ (from Ref. [42•], with permission). High-spatialresolution $\mathbf{d}$ morphologic, e GagCEST, and $\mathbf{f}^{23} \mathrm{Na} \mathrm{MR}$ images of the knee joint cartilage of a patient $(26.4$ years old) who underwent matrixassociated autologous chondrocyte transplantation (MACT) in the lateral femoral condyle (from Ref. [43] with permission.)

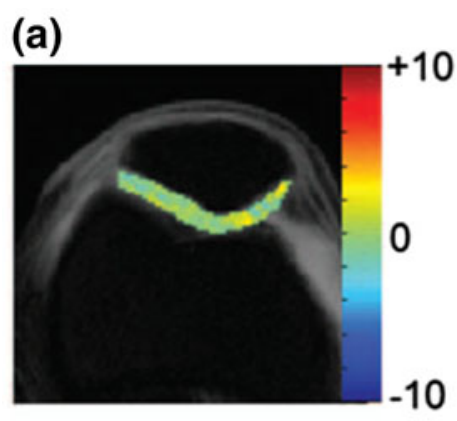

(b)

(d)

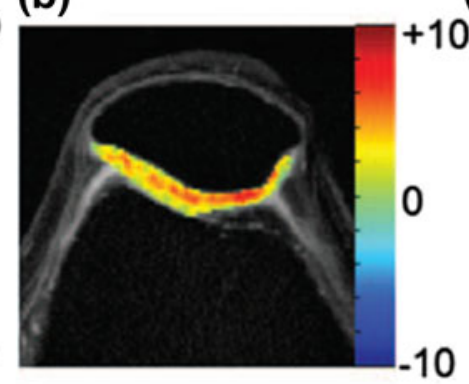

(e)

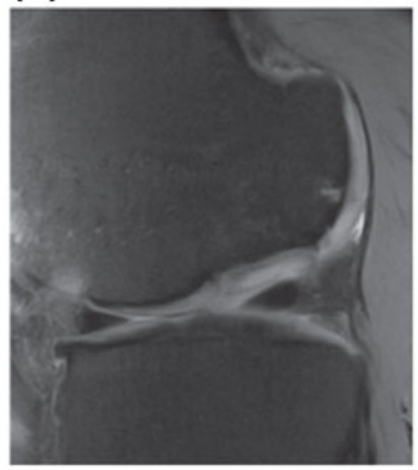

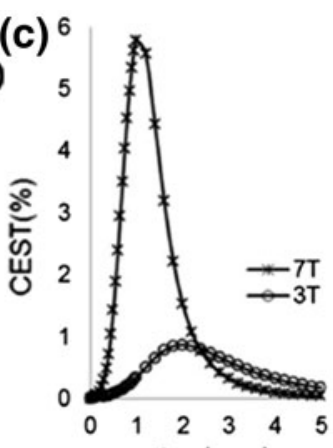

(f)

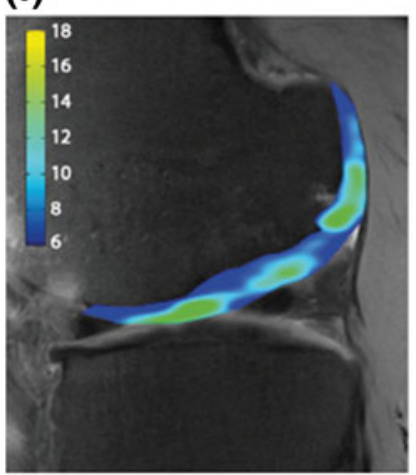

(a)

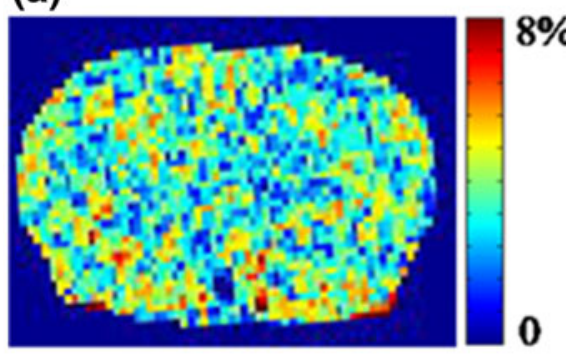

Proton MRS

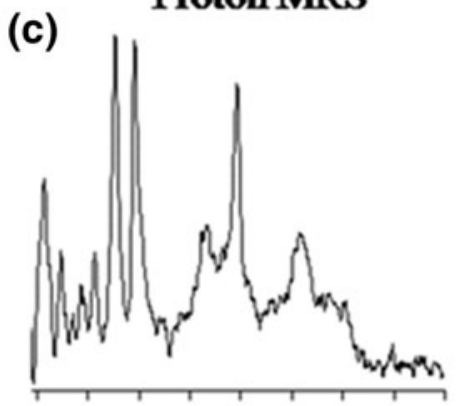

$\begin{array}{lllllllll}4 & 3.5 & 3 & 2.5 & 2 & 1.5 & 1 & 0.5 & 0\end{array}$

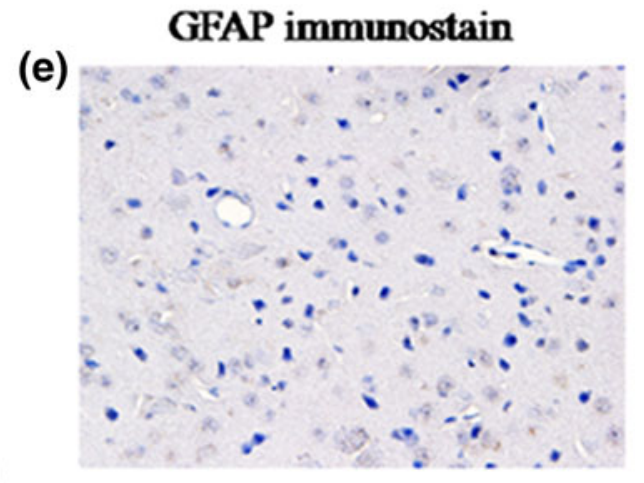

(b)

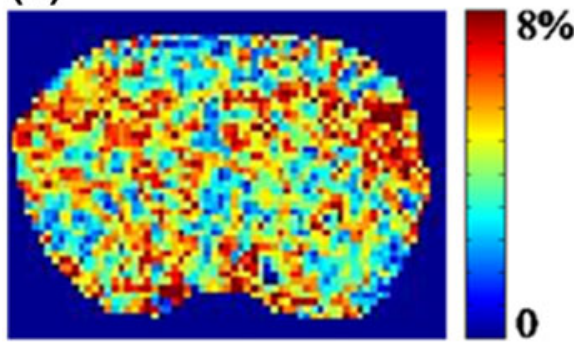

(d)

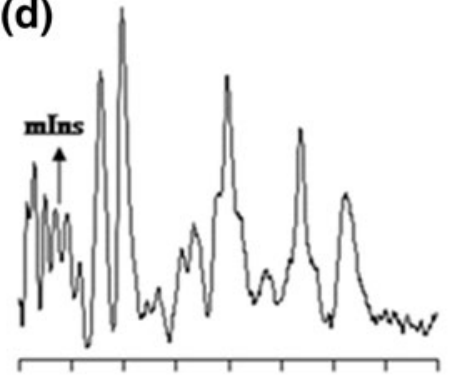

$\begin{array}{lllllllll}4 & 3.5 & 3 & 2.5 & 2 & 1.5 & 1 & 0.5 & 0\end{array}$ (f)

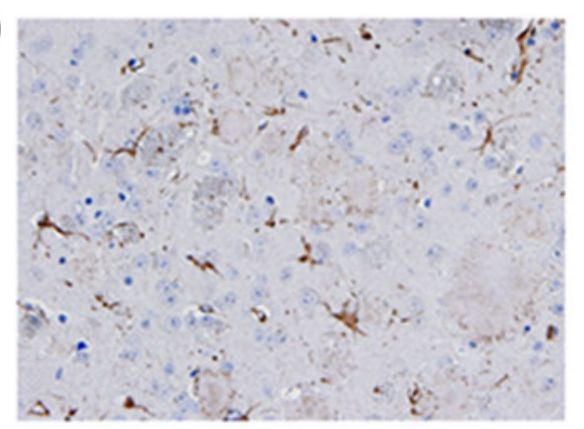

Fig. 4 MICEST maps show the distribution of myoinositol in the brain of a a 20-month-old wild-type mouse and a b 20-month-old APP-PS1 transgenic mouse model of Alzheimer's disease (AD). Higher MICEST contrast is depicted in the AD brain compared to the WT mouse. ${ }^{1} \mathrm{H}$ MRS spectrum shows that compared to the $\mathbf{c}$ wild type mouse, there was an increase in the myoinositol peak amplitude in the d AD mouse. GFAP immunostain of brain slices from e WT and f APP-PS1 mice show higher expression of GFAP in the APP-PS1 mouse than the WT mouse. This signifies higher glial cell proliferation/activation in APP-PS1 mice (from Ref. [48], with permission.) 
In general, $-\mathrm{OH}$ groups of many metabolites, such as the one described above, resonate at around $1 \mathrm{ppm}$ (0.6-1.5 ppm) down field from water and have exchange rates in the range of 500-1,500 $\mathrm{s}^{-1}$. These exchange rates typically do not satisfy the condition of slow to intermediate exchange [Eq. (1)] on the NMR time scale at lower fields such as $1.5 \mathrm{~T}$ and $3 \mathrm{~T}$. In addition, lower frequency separation from water and the requirement of relatively high saturation power lead to huge direct saturation effects that decrease the sensitivity of CEST. However, as discussed in this section, these studies can be performed at higher fields $(\geq 7 \mathrm{~T})$ with improved sensitivity and have been demonstrated in preclinical as well as in clinically relevant applications.

\section{Amine $\left(-\mathrm{NH}_{2}\right)$ Protons}

Amine protons from free amino acids or protein and peptide side chains are another important class of endogenous CEST agents. Endogenous metabolites with exchangeable amine group protons and exchange rates suitable for CEST imaging include Glu and $\mathrm{Cr}$.

Glutamate is the major excitatory neurotransmitter in the central nervous system (CNS). It was recently demonstrated that Glu exhibits a $\mathrm{pH}$ - and concentration-dependent CEST effect (GluCEST) between its amine group, observed at $\sim 3.0$ ppm downfield from water, and bulk water [52•]. Glu$\mathrm{CEST}_{\text {asym }}$ obtained from a rat brain following middle cerebral artery occlusion (MCAO) induced stroke showed significant differences between ipsilateral and contralateral sides (Fig. 5a-c). The ipsilateral side demonstrated a $\sim 100 \%$ increase in GluCEST asym $_{\text {at }} 4.5 \mathrm{~h}$ after MCAO (Fig. 5d). The elevated GluCEST ${ }_{\text {asym }}$ in the ipsilateral side of MCAO model was determined to be predominantly due to decreased $\mathrm{pH}$.
Additionally, intravenous Glu injected in a rat brain tumor model with a compromised blood brain barrier led to an elevation of GluCEST asym $_{\text {around the tumor while no changes }}$ were seen in the normal appearing tissue. Finally, GluCEST maps of the brain in healthy human subjects showed a distinct white matter, gray matter distribution and demonstrated the feasibility of mapping relative changes in Glu concentration as well as $\mathrm{pH}$ in vivo. For validation, a strong correlation was observed between the GluCEST ${ }_{\text {asym }}$ ratio from GM and WM ROIs $(1.6 \pm 0.2)$ and the measured Glu concentration ratio from ${ }^{1} \mathrm{H}$ magnetic resonance spectroscopy (MRS) data from the same ROIs $(1.6 \pm 0.1)$. Recent studies have used this approach to investigate changes in Glu in a rat model of Alzheimer's disease [53] as well as to map Glu in the spinal cord [54]. This technique has the ability to provide new insights into the role of Glu in the CNS as well as serve as a biomarker for diagnosis and treatment of CNS disorders. Given the excellent sensitivity to $\mathrm{pH}$, with proper calibration it may be possible to use GluCEST for determining $\mathrm{pH}$.

Creatine $(\mathrm{Cr})$ is another important metabolite that has exchangeable amine protons. Cr plays an essential role in the storage and transmission of phosphate-bound energy. During skeletal muscle exercise, phosphocreatine ( $\mathrm{PCr}$ ) is depleted to maintain the adenosine triphosphate (ATP) supply leading to an increase $\mathrm{Cr}$ concentration and thus $\mathrm{Cr}$ can be used as a marker of muscle energetics. Cr exhibits a CEST effect (CrCEST) between its amine $\left(-\mathrm{NH}_{2}\right)$ and bulk water protons [55-57]. $\mathrm{Cr}$ amine protons have an exchange site at $\sim 1.8$ ppm from water and the CEST effect from $\mathrm{Cr}$ is linearly proportional to the $\mathrm{Cr}$ concentration in the physiological $\mathrm{pH}$ range. Furthermore, this CEST effect from $\mathrm{Cr}$ can be isolated from the other metabolites of the creatine kinase reaction (PCr, ATP, ADP) based on their exchange rate differences [57]. ${ }^{31} \mathrm{P}$ MRS has been used
Fig. 5 GluCEST $_{\text {asym }}$ maps of an ischemic rat brain model. a Rat brain anatomic proton image. b, $\mathbf{c}$ The GluCEST ${ }_{\text {asym }}$ maps of the rat brain acquired at 1 and $4.5 \mathrm{~h}$ following the induction of stroke. d The plot

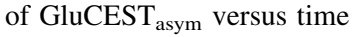
after MCAO at regions of interest within the rectangular areas shown in c. In the ipsilateral side GluCEST asym $_{\text {is }}$ almost doubled at $4.5 \mathrm{~h}$ after occlusion. e The GluCEST asym $_{\text {}}$ plots from the contralateral side (blue curve) and ipsilateral side (red curve) (from Ref. [52•], with permission.) (a)
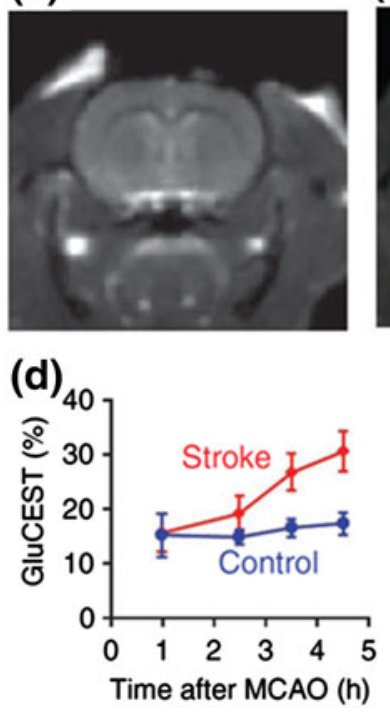

(b)

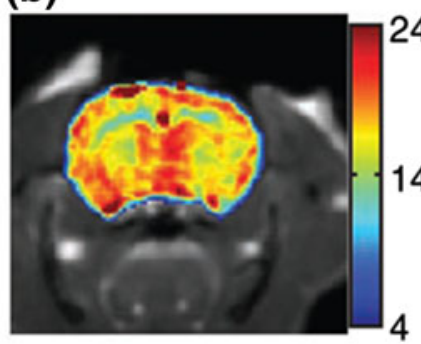

(c)

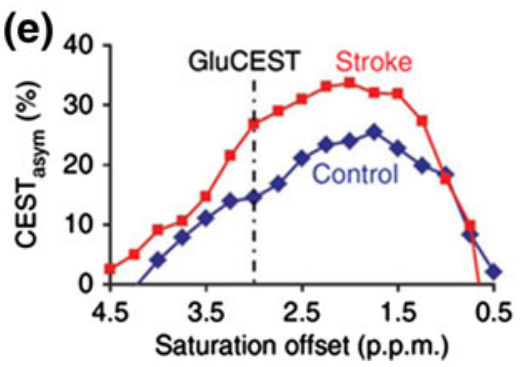


(a)
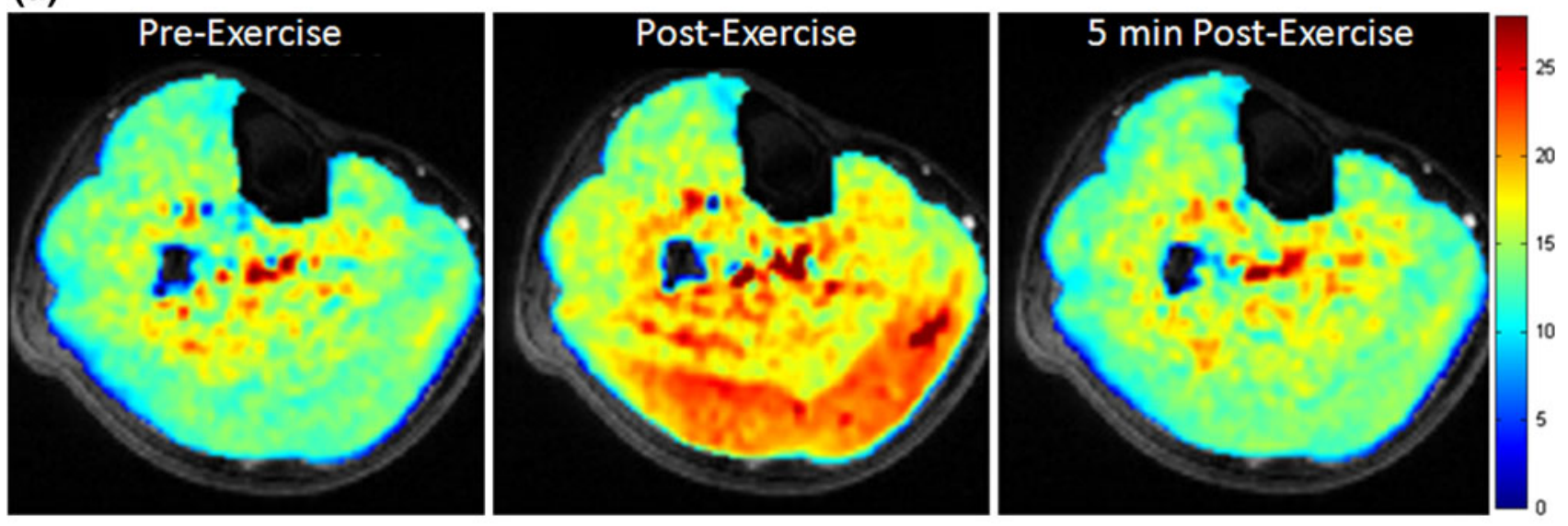

(b)

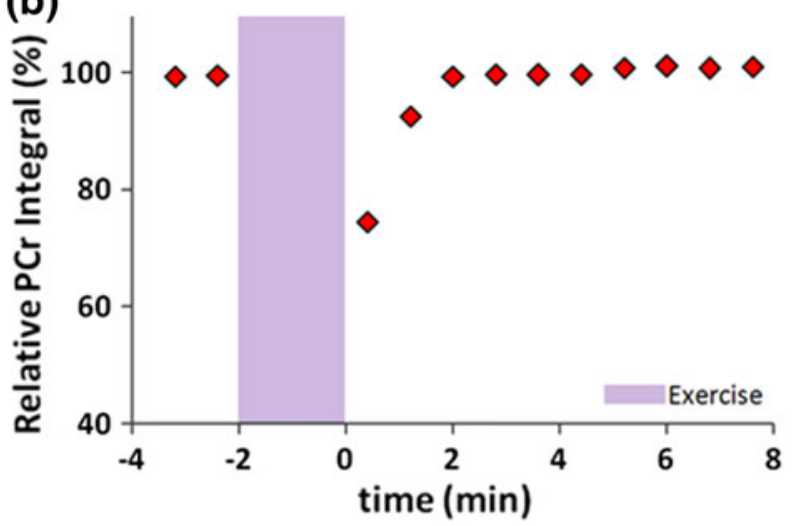

Fig. 6 a $\mathrm{CrCEST}_{\text {asym }}$ maps of a human lower leg before and after plantar flexion exercise. b The plot of ${ }^{31} \mathrm{P}$ MRS PCr peak integral as a function of time before and after exercise. $\mathbf{c}$ The plot of the average

extensively to study oxidative metabolism of skeletal muscle and is able to relay information about the concentrations of $\mathrm{PCr}, \mathrm{Pi}$, as well as muscle $\mathrm{pH}$ [58-61]. However, ${ }^{31} \mathrm{P}$ MRS, like all spectroscopy techniques, suffers from poor spatial resolution as well as low sensitivity due the low gyromagnetic ratio of ${ }^{31} \mathrm{P}$. CrCEST is able to image $\mathrm{Cr}$ distribution in muscle and thus may provide information about creatine kinase kinetics with high spatial resolution. The feasibility of using this technique to spatially mapping free $\mathrm{Cr}$ changes in skeletal muscle in healthy volunteers has been demonstrated at 7T [62]. Mild plantar flexion exercise led to an increase in CrCEST that was well localized to the posterior compartment of the lower leg and, as expected, exponentially recovered back to baseline in $\sim 2 \mathrm{~min}$ (Fig. 6a). CrCEST results were compared with ${ }^{31} \mathrm{P}$ MRS results showing good agreement in the recovery kinetics of CrCEST and PCr signal following exercise (Fig. 6b, c). In addition to skeletal muscle, this approach has also been applied to cardiac muscle energetics [63]. Future studies using this approach may provide new insights into muscle

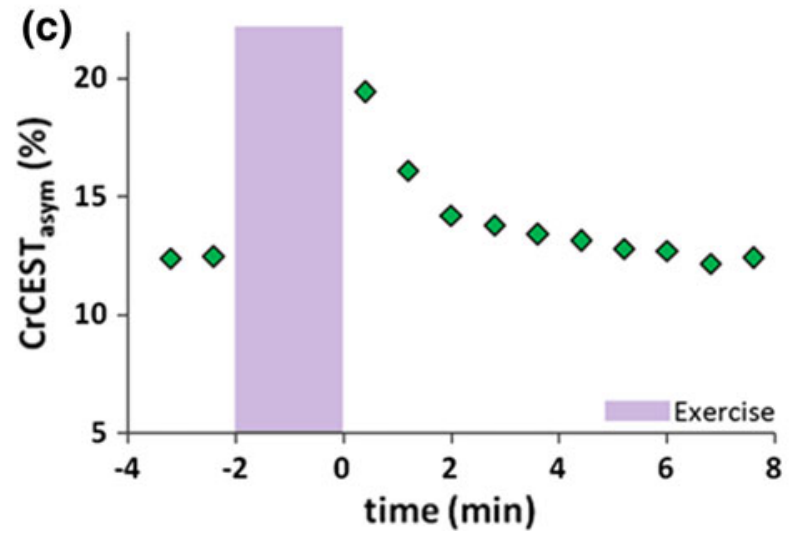

$\mathrm{CrCEST}_{\text {asym }}$ as a function of time in a region of interest selected to correlate to the depth of penetration of the ${ }^{31} \mathrm{P}$ MRS surface coil (unpublished results from author's laboratory)

energetics and can serve as a tool for the diagnosis and treatment of skeletal muscle and cardiac disorders.

Additionally, amine-proton exchange has been studied collectively (APEX) by applying a high amplitude and shorter duration saturation pulse at $\Delta \omega= \pm 2.5 \mathrm{ppm}$ [64]. Ischemia induced by cardiac arrest or MCAO, resulted in a decrease in APT contrast but significant increase in APEX contrast. However, this method lacks specificity and only tracks local $\mathrm{pH}$ or amine proton concentration changes.

Compared to amide, amine protons tend to have faster exchange rates. While this allows for higher saturation transfer efficiency, a higher $B_{1}$ amplitude is required in order to achieve saturation, which increases direct water saturation effects and may be limited by Food and Drug Administration (FDA) Specific Absorption Rate (SAR) regulations. Typically, the faster exchange rates of amine protons do not satisfy the slow to intermediate exchange condition [Eq. (1)] at low fields $(\leq 3 \mathrm{~T})$ and as a result, amine CEST studies have to be performed at ultrahigh fields $(\geq 7 \mathrm{~T})$. 


\section{CEST Imaging of $\mathrm{pH}$}

$\mathrm{pH}$ is an important marker of many disease processes and pathologies including cancer and stroke. Current noninvasive approaches to measure $\mathrm{pH}$ in vivo rely on ${ }^{1} \mathrm{H}$ and ${ }^{31} \mathrm{P}$ MRS [65]. However, the poor spatial resolution and scan time limitations have limited their application in the clinic. On the other hand, the direct effect of $\mathrm{pH}$ on chemical exchange rate makes CEST an ideal technique to assess change in $\mathrm{pH}$ in vivo with high spatial resolution. As a result, CEST imaging has been used to study and attempt to quantify changes in $\mathrm{pH}$ $[55,66,67]$. In fact, image contrast due to changes in $\mathrm{pH}$ has been utilized in a significant amount of APT studies.

CEST based $\mathrm{pH}$ quantification has its own challenges. In an in vitro setting where other factors can be controlled for, several studies have developed algorithms to measure and accurately quantify differences in $\mathrm{pH}$ [68]. However, CEST contrast depends on several parameters including labile proton concentration, temperature, water content, the $T_{1}$ of water, saturation parameters as well as any other factors which affect the chemical environment of the exchanging protons. This makes in vivo $\mathrm{pH}$ quantification significantly more challenging as accounting for all of these factors in vivo is rather difficult. An alternate strategy is to use a CEST agent with two exchanging sites, which can be used as an internal reference to control for many of these confounds. By using a CEST agent with two exchange sites, the ratio of the CEST asymmetry at each exchange site will
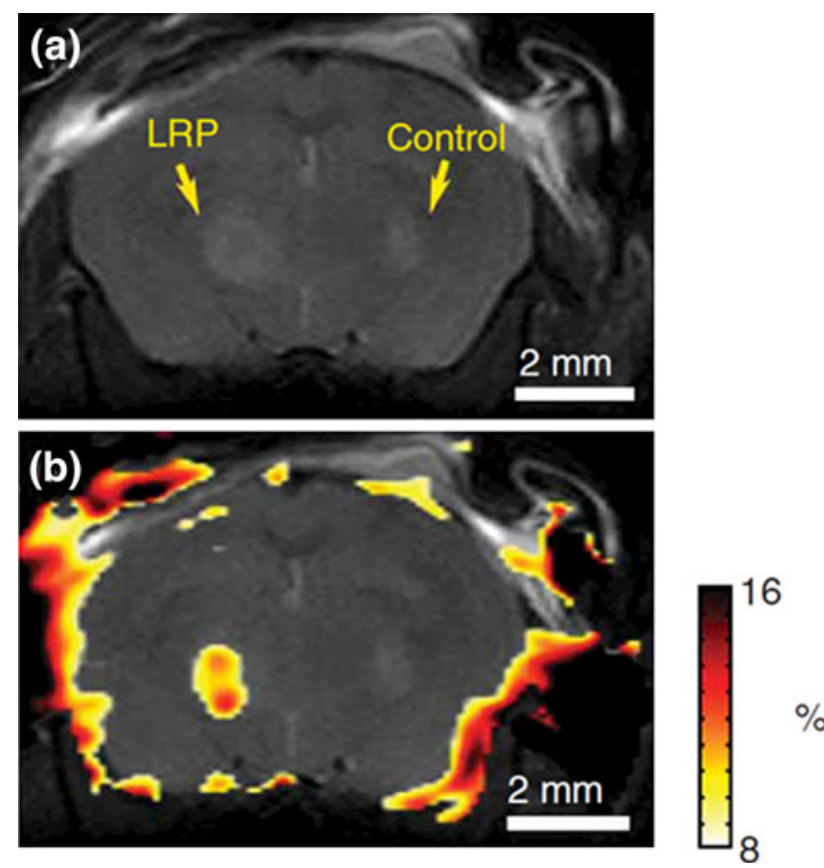

Fig. 7 In vivo imaging of lysine rich protein (LRP). a Anatomical image and b CEST signal intensity-difference map overlaid on the anatomical image distinguishes between LRP-expressing and control xenografts (from Ref. [69], with permission.) vary with the ratio of exchange rates, and can thus be used for $\mathrm{pH}$ calibration [66]. However, this technique was only validated in vitro and has not been applied to in vivo endogenous $\mathrm{pH}$ measurement studies.

\section{Exogenous Contrast Agents}

After its discovery as a mechanism of contrast in MRI, CEST was discussed as a possible mechanism for exogenous contrast agents [22]. Unlike most MRI contrast agents, which alter water proton $T_{1}$ or $T_{2}$, these agents reduce the water proton signal through a chemical exchange site on the contrast agent. A major advantage of using saturation transfer over conventional contrast agents is that the agents can be switched on and off depending on the saturation frequency. CEST exogenous agents have several advantages. First, they can be designed to look at a particular metabolite or receptor. These agents can be polymerized to increase the number of exchange sites per mole and can possess multiple exchange sites. One of the applications of exogenous CEST agents is as reporter genes, which could provide information about the distribution and viability of targeted cells in vivo. Current MR techniques rely on paramagnetic or superparamagnetic substrates to shorten $T_{1}$ and $T_{2}$ and cannot be turned off. Recently, oligonucleotides were designed that encoded lysine rich protein (LRP) (200 lysine residues; $32 \mathrm{kDa}$ ), which were cloned into a mammalian expression vector [69]. Poly-L-lysine has amide protons, which exchange with water protons to create CEST contrast. The vector was transfected into $9 \mathrm{~L}$ rat glioma cells. CEST asymmetry maps were then able to distinguish LRP-expressing tumors from control tumors (Fig. 7).

While exogenous CEST agents have some advantages, as with all contrast agents, they must be introduced into the body, usually through intravascular injection, which may increase scan time to allow the contrast agent to perfuse into the tissue. Additionally, prior to in vivo human studies with these agents, including paramagnetic agents, their potential toxicity has to be addressed.

\section{Technical Considerations}

The CEST effect depends on several factors such as field strength $\left(B_{0}\right)$, concentration of metabolite with exchanging spins, exchange rate, $B_{0}$ and $B_{1}$ field homogeneities, $T_{1}$ of water protons, RF saturation pulse duration and amplitude. Thus in measuring the CEST effect from a given metabolite all these factors have to be optimized and accounted for.

\section{Field Homogeneity}

Static magnetic field $\left(B_{0}\right)$ and RF field $\left(B_{1}\right)$ inhomogeneities present a challenge for CEST imaging. This is 
particularly significant at ultrahigh magnetic fields, where the effects of these inhomogeneities are magnified [21]. $B_{0}$ field inhomogeneities lead to a shift in the water resonance frequency that results in asymmetric direct water saturation effects and as a result artificial CEST effects in asymmetry analysis. Even small shifts in field inhomogeneity may lead to large errors in the measured CEST asymmetry. $B_{1}$ inhomogeneity on the other hand results an increase or decrease in the applied RF. This leads to either a reduction of saturation efficiency or an increase in direct water saturation effects, which will create inaccuracies in the CEST asymmetry maps. Several methods have been developed for correction of $B_{0}$ and $B_{1}$ inhomogeneities [28, 70, 71]. Accurate correction of field inhomogeneities is essential to precise CEST asymmetry measurements.

\section{CEST Pulse Sequences}

In general, low-power, long-duration rectangular saturation pulses are employed in phantom and animal model studies. However, due to clinical scanner limitations, trains of Gaussian or Hanning windowed short duration pulses separated by short delays are employed. Currently, most applications of CEST utilize a single slice readout. CEST requires acquisition at multiple saturation frequencies with long repetition times (TR) to allow for relaxation. To address this issue, new multislice and three-dimensional (3D) acquisition techniques have emerged to decrease scan times. One approach is to insert a short frequency-selective saturation pulse between spatially selective excitation pulses in a standard 2D gradient-echo (GRE) sequence [72]. As the TR of GRE readout pulses is much less than $T_{1}$, this leads to an additive effect of saturation pulses for slowly exchanging spins and thus can use an interleaved, multi-slice readout. Another method utilizes a long primary saturation pulse to generate CEST contrast and repetitive short secondary saturation pulses immediately after the image acquisition so as to maintain the steady state CEST contrast for multi-slice acquisition and signal averaging [73]. Finally, a 3D gradient and spin-echo (GRASE) image readout has been implemented in combination with a multichannel coil that allows for parallel imaging techniques to obtain a $z$-spectrum ( 26 frequencies) of the entire brain in less than $10 \mathrm{~min}$ [74]. All of these methods rely on steady state CEST contrast and as a result may not be optimal for faster exchanging spins. Development of faster, multislice or 3D CEST techniques is important to translating CEST imaging to more clinical applications.

\section{Line-Fitting Methods}

In order to address the many confounders of the CEST effects including NOE effects and MTR asymmetry several methods have also been developed that utilize z-spectral fitting for computing the CEST effect. One method utilizes multiple saturation frequency offsets centered around the z-spectral dips of amide protons, MT asymmetry and NOE in order to isolate each respective $\mathrm{z}$-spectral contribution [75]. However, this technique requires that a narrow peak be observed in the z-spectra and thus has limited applicability at lower fields and faster exchanging spins. Another approach fits z-spectral data using Lorentzian functions corresponding to direct saturation (DS), magnetization transfer (MT) and CEST components using either linear or probabilistic combinations [76]. While these methods show promise for decoupling the confounding contributions to the CEST effect, further work is necessary to assess their in vivo accuracy.

Alternative Exchange-Based Approaches

In addition to the conventional method of measuring $\mathrm{CEST}_{\text {asym }}$ described by Eq. (2), several other methods have been developed for exchange transfer MRI. These include frequency-labeled exchange transfer (FLEX) [77], combining chemical exchange saturation transfer and $T_{1 \rho}$ magnetization preparations (CESTrho) [78], length and offset varied saturation (LOVARS) [79], two-frequency RF irradiation [80], chemical exchange rotation transfer (CERT) [81] as well as others. These methods may further advance exchange based MRI, but need further characterization in in vivo applications.

\section{Conclusion}

Endogenous CEST applications show promise to use MRI as a noninvasive, nonionizing tool for molecular imaging. Recent work has identified and imaged in vivo several endogenous metabolites with exchangeable protons. Several studies have demonstrated the feasibility of implementing these methods both in preclinical models as well as in human studies. These endogenous compounds can be exploited as biomarkers for diagnosis and characterization of diseases such as cancer, neurological or psychiatric diseases, stroke, osteoarthritis and various skeletal muscle disorders as well as in treatment monitoring. Recent advances in CEST techniques have rapidly grown the field and further developments in improving the acquisition speed and spatial coverage, as well as techniques to enhance the specificity of the methods will enable widespread translation into the clinical setting.

Acknowledgments This work was supported by a NIBIB supported resources center Grants P41-EB015893, P41-EB015893-S1 and NIH Grants 1R21-DA032256-01 and T32EB009384. A pilot grant from TBIC of ITMAT of the University of Pennsylvania. 
Disclosure No potential conflicts of interest relevant to this article were reported.

\section{References}

Papers of particular interest, published recently, have been highlighted as:

- Of importance

1. Ametamey SM, Treyer V, Streffer J, et al. Human PET studies of metabotropic glutamate receptor subtype 5 with C-11-ABP688. J Nucl Med. 2007;48(2):247-52.

2. Dunn WB, Ellis DI. Metabolomics: current analytical platforms and methodologies. TrAC Trends Anal Chem. 2005;24(4):285-94.

3. Burt CT, Glonek T, Barany M. Analysis of phosphate metabolites, intracellular $\mathrm{pH}$, and state of adenosine-triphosphate in intact muscle by phosphorus nuclear magnetic-resonance. J Biol Chem. 1976;251(9):2584-91.

4. Kreis R, Ernst T, Ross BD. Absolute quantitation of water and metabolites in the human brain. 2. Metabolite concentrations. J Magn Reson Ser B. 1993;102(1):1-19.

5. Provencher SW. Estimation of metabolite concentrations from localized in vivo proton NMR spectra. Magn Reson Med. 1993; 30(6):672-9.

6. Caravan P. Strategies for increasing the sensitivity of gadolinium based MRI contrast agents. Chem Soc Rev. 2006;35(6):512-23.

7. Bulte JWM, Kraitchman DL. Iron oxide MR contrast agents for molecular and cellular imaging. NMR Biomed. 2004;17(7):484-99.

8. Arnold JT, Packard ME. Variations in absolute chemical shift of nuclear induction signals of hydroxyl groups of methyl and ethyl alcohol. J Chem Phys. 1951;19(12):1608-9.

9. Gutowsky HS, Saika A. Dissociation, chemical exchange, and the proton magnetic resonance in some aqueous electrolytes. J Chem Phys. 1953;21(10):1688-94.

10. Forsen S, Hoffman R. Study of moderately rapid chemical exchange reactions by means of nuclear magnetic double resonance. J Chem Phys. 1963;39(11):2892-901.

11. Wolff S, Balaban R. NMR imaging of labile proton-exchange. J Magn Reson. 1990;86(1):164-9.

12. Guivel-Scharen V, Sinnwell T, Wolff S, Balaban R. Detection of proton chemical exchange between metabolites and water in biological tissues. J Magn Reson. 1998;133(1):36-45.

13. Dagher A, Aletras A, Choyke P, Balaban R. Imaging of urea using chemical exchange-dependent saturation transfer at $1.5 \mathrm{~T}$. J Magn Reson Imaging. 2000;12(5):745-8.

14. Zhang SR, Merritt M, Woessner DE, et al. PARACEST agents: modulating MRI contrast via water proton exchange. Acc Chem Res. 2003;36(10):783-90.

15. Woods M, Donald EWC, Sherry AD. Paramagnetic lanthanide complexes as PARACEST agents for medical imaging. Chem Soc Rev. 2006;35(6):500-11.

16. Aime S, Crich SG, Gianolio E, et al. High sensitivity lanthanide(III) based probes for MR-medical imaging. Coord Chem Rev. 2006;250(11-12):1562-79.

17. Terreno E, Castelli DD, Aime S. Encoding the frequency dependence in MRI contrast media: the emerging class of CEST agents. Contrast Media Mol Imaging. 2010;5(2):78-98.

18. Hancu I, Dixon WT, Woods M, et al. CEST and PARACEST MR contrast agents. Acta Radiol. 2010;51(8):910-23.

19. Sherry A, Woods M. Chemical exchange saturation transfer contrast agents for magnetic resonance imaging. Annu Rev Biomed Eng. 2008;10:391-411.
20. Zhou J, van Zijl P. Chemical exchange saturation transfer imaging and spectroscopy. Prog Nucl Magn Reson Spectrosc. 2006;48(2-3):109-36.

21. van Zijl PCM, Yadav NN. Chemical exchange saturation transfer (CEST): what is in a name and what isn't? Magn Reson Med. 2011;65(4):927-48.

22. Ward K, Aletras A, Balaban R. A new class of contrast agents for MRI based on proton chemical exchange dependent saturation transfer (CEST). J Magn Reson. 2000;143(1):79-87.

23. Liu G, Gilad AA, Bulte JWM, et al. High-throughput screening of chemical exchange saturation transfer MR contrast agents. Contrast Media Mol Imaging. 2010;5(3):162-70.

24. Sun P, van Zijl P, Zhou J. Optimization of the irradiation power in chemical exchange dependent saturation transfer experiments. J Magn Reson. 2005;175(2):193-200.

25. Woessner D, Zhang S, Merritt M, Sherry A. Numerical solution of the Bloch equations provides insights into the optimum design of PARACEST agents for MRI. Magn Reson Med. 2005;53(4): 790-9.

26. Liepinsh E, Otting G. Proton exchange rates from amino acid side chains-implications for image contrast. Magn Reson Med. 1996;35(1):30-42.

27. Zhou J, Payen J, Wilson D, et al. Using the amide proton signals of intracellular proteins and peptides to detect $\mathrm{pH}$ effects in MRI. Nat Med. 2003;9(8):1085-90.

28. Zhou J, Lal B, Wilson D, et al. Amide proton transfer (APT) contrast for imaging of brain tumors. Magn Reson Med. 2003; 50(6):1120-6.

29. Zhou J, Wilson D, Sun P, et al. Quantitative description of proton exchange processes between water and endogenous and exogenous agents for WEX, CEST, and APT experiments. Magn Reson Med. 2004;51(5):945-52.

30. Hua J, Jones C, Blakeley J, et al. Quantitative description of the asymmetry in magnetization transfer effects around the water resonance in the human brain. Magn Reson Med. 2007;58(4): 786-93.

31. Jones C, Schlosser M, van Zijl P, et al. Amide proton transfer imaging of human brain tumors at 3T. Magn Reson Med. 2006;56(3):585-92.

32. Zhou J, Blakeley JO, Hua J, et al. Practical data acquisition method for human brain tumor amide proton transfer (APT) imaging. Magn Reson Med. 2008;60(4):842-9.

33. Wen Z, Hu S, Huang F, et al. MR imaging of high-grade brain tumors using endogenous protein and peptide-based contrast. Neuroimage. 2010;51(2):616-22.

34. - Zhou J, Tryggestad E, Wen Z, et al. Differentiation between glioma and radiation necrosis using molecular magnetic resonance imaging of endogenous proteins and peptides. Nat Med. 2011;17(1):130-U308. Demonstrated the feasibility of using APT CEST MRI to differentiate active tumors from radiation necrosis.

35. Dula AN, Asche EM, Landman BA, et al. Development of chemical exchange saturation transfer at 7T. Magn Reson Med. 2011;66(3):831-8.

36. Schmitt B, Zamecnik P, Zaiss M, et al. A new contrast in MR mammography by means of chemical exchange saturation transfer (CEST) imaging at 3 Tesla: preliminary results. Rofo Fortschr Rontg. 2011;183(11):1030-6.

37. Jia G, Abaza R, Williams JD, et al. Amide proton transfer MR imaging of prostate cancer: a preliminary study. J Magn Reson Imaging. 2011;33(3):647-54.

38. Dula A, Dortch R, Landman B, et al. Application of chemical exchange saturation transfer (CEST) imaging to examine amide proton transfer (APT) in the spinal cord at 3T. Proceedings of the 19th Annual Meeting of ISMRM, Montreal; 2011. p. 407.

39. - van Zijl PCM, Jones CK, Ren J, et al. MR1 detection of glycogen in vivo by using chemical exchange saturation transfer 
imaging (glycoCEST). Proc Natl Acad Sci USA 2007;104(11): 4359-4364. First study to detect the CEST effect from glycogen in vitro and in vitro as well as in vivo in animal studies of the liver at $4.7 T$.

40. Ling W, Regatte R, Navon G, Jerschow A. Assessment of glycosaminoglycan concentration in vivo by chemical exchangedependent saturation transfer (gagCEST). Proc Natl Acad Sci USA. 2008;105(7):2266-70.

41. Rizkalla G, Reiner A, Bogoch E, Poole AR. Studies of the articular-cartilage proteoglycan aggrecan in health and osteoarthritis-evidence for molecular heterogeneity and extensive molecular-changes in disease. J Clin Invest. 1992;90(6):2268-77.

42. - Singh A, Haris M, Cai K, et al. Chemical exchange saturation transfer magnetic resonance imaging of human knee cartilage at 3T and 7T. Magn Reson Med. 2012;68(2):588-594. Showed that correction of $B_{O}$ inhomogeneities lead to negligible GagCEST contrast at 3T. Also demonstrated that moving to 7T, makes GagCEST mapping possible in the knee.

43. Schmitt B, Zbyn S, Stelzeneder D, et al. Cartilage quality assessment by using glycosaminoglycan chemical exchange saturation transfer and Na-23 MR imaging at 7T. Radiology. 2011; 260(1):257-64.

44. Kim M, Chan Q, Anthony M-P, et al. Assessment of glycosaminoglycan distribution in human lumbar intervertebral discs using chemical exchange saturation transfer at 3T: feasibility and initial experience. NMR Biomed. 2011;24(9):1137-44.

45. Saar G, Zhang B, Ling W, et al. Assessment of glycosaminoglycan concentration changes in the intervertebral disc via chemical exchange saturation transfer. NMR Biomed. 2012; 25(2):255-61.

46. Schmitt B, Brix M, Hofstaetter J, et al. Evaluation of the dependency of glycosaminoglycan (gag) chemical exchange saturation transfer (gagCEST) imaging on cartilage gag content in the ankle at 3T. Osteoarthritis Cartilage. 2012;20:S222.

47. - Haris M, Cai K, Singh A, et al. In vivo mapping of brain myoinositol. NeuroImage 2011;54(3):2079-2085. Novel work that characterized the CEST effect from Myo-inositol and demonstrated the feasibility of MICEST Brain mapping.

48. Haris M, Singh A, Cai K, Nath K, et al. MICEST: a potential tool for non-invasive detection of molecular changes in Alzheimer's disease. J Neurosci Methods. 2013;212(1):87-93.

49. Walker-Samuel S, Johnson P, Pedley B, et al. Assessment of tumour glucose uptake using gluco-CEST. Proceedings of the 19th annual meeting of ISMRM, Montreal; 2011. p. 962.

50. Chilakapati S, Reddy S, Haris M, et al. Characterization of CEST effect from glucose in vitro. Proceedings of the 20th annual meeting of ISMRM, Melbourne; 2012. p. 2335.

51. Chan KWY, McMahon MT, Kato Y, et al. Natural D-glucose as a biodegradable MRI contrast agent for detecting cancer. Magn Reson Med. 2012;68(6):1764-73.

52. - Cai K, Haris M, Singh A, et al. Magnetic resonance imaging of glutamate. Nat Med 2012;18(2):302-306. First work to demonstrate the feasibility of mapping the CEST effect from glutamate in vivo. Also characterized the GluCEST dependence on $\mathrm{pH}$ and concentration in vivo in animal models.

53. Haris M, Nath K, Cai K, et al. Imaging of glutamate neurotransmitter alterations in Alzheimer's disease. NMR Biomed. 2012. doi:10.1002/nbm.2875.

54. Kogan F, Singh A, Haris M, et al. Imaging of glutamate in the spinal cord using chemical exchange saturation transfer (CEST) at 7 T. Proceedings of the 20th annual meeting of ISMRM, Melbourne; 2012. p. 737.

55. Sun P, Benner T, Kumar A, Sorensen A. Investigation of optimizing and translating $\mathrm{pH}$-sensitive pulsed-chemical exchange saturation transfer (CEST) imaging to a 3T clinical scanner. Magn Reson Med. 2008;60(4):834-41.
56. Singh A, Haris M, Cai K, et al. Chemical exchange transfer imaging of creatine. Proceedings of the 19th annual meeting of ISMRM, Montreal; 2011. p. 2771.

57. Haris M, Nanga RPR, Singh A, et al. Exchange rates of creatine kinase metabolites: feasibility of imaging creatine by chemical exchange saturation transfer MRI. NMR Biomed. 2012. doi: 10.1002/nbm.2792.

58. Hoult DI, Busby SJW, Gadian DG, et al. Observation of tissue metabolites using P-31 nuclear magnetic-resonance. Nature. 1974;252(5481):285-7.

59. Dawson MJ, Gadian DG, Wilkie DR. Muscular fatigue investigated by phosphorus nuclear magnetic-resonance. Nature. 1978; 274(5674):861-6.

60. Ingwall JS. Phosphorus nuclear magnetic-resonance spectroscopy of cardiac and skeletal-muscles. Am J Physiol. 1982;242(5): H729-44.

61. Kemp GJ, Meyerspeer M, Moser E. Absolute quantification of phosphorus metabolite concentrations in human muscle in vivo by P-31 MRS: a quantitative review. NMR Biomed. 2007;20(6): 555-65.

62. Haris M, Cai K, Singh A, et al. Detection of exercised induced changes in creatine level in human calf muscles through CEST: a preliminary study. Proceedings of the 20th annual meeting of ISMRM, Melbourne; 2012. p. 2342.

63. Haris M, Singh A, Cai K, et al. Assessment of chemical exchange saturation transfer effects in myocardial tissue at 7T. Proceedings of the 19th annual meeting of ISMRM, Montreal; 2011. p. 22.

64. Jin T, Wang P, Zong X, Kim S-G. Magnetic resonance imaging of the Amine-Proton EXchange (APEX) dependent contrast. NeuroImage. 2012;59(2):1218-27.

65. Sahlin K, Harris RC, Nylind B, Hultman E. Lactate content and $\mathrm{pH}$ in muscle samples obtained after dynamic exercise. Pflugers Arch EJP. 1976;367(2):143-9.

66. Ward K, Balaban R. Determination of $\mathrm{pH}$ using water protons and chemical exchange dependent saturation transfer (CEST). Magn Reson Med. 2000;44(5):799-802.

67. Jokivarsi K, Grohn H, Grohn O, Kauppinen R. Proton transfer ratio, lactate, and intracellular $\mathrm{pH}$ in acute cerebral ischemia. Magn Reson Med. 2007;57(4):647-53.

68. Sun P, Sorensen A. Imaging $\mathrm{pH}$ using the chemical exchange saturation transfer (CEST) MRI: correction of concomitant RF irradiation effects to quantify CEST MRI for chemical exchange rate and $\mathrm{pH}$. Magn Reson Med. 2008;60(2):390-7.

69. Gilad AA, McMahon MT, Walczak P, et al. Artificial reporter gene providing MRI contrast based on proton exchange. Nat Biotechnol. 2007;25(2):217-9.

70. Kim M, Gillen J, Landman B, et al. Water saturation shift referencing (WASSR) for chemical exchange saturation transfer (CEST) experiments. Magn Reson Med. 2009;61(6):1441-50.

71. Singh A, Cai K, Haris M, et al. On B1 inhomogeneity correction of in vivo human brain glutamate chemical exchange saturation transfer contrast at 7T. Magn Reson Med. 2012. doi:10.1002/ mrm.24290.

72. Dixon W, Hancu I, Ratnakar S, et al. A multislice gradient echo pulse sequence for CEST imaging. Magn Reson Med. 2010; 63(1):253-6.

73. Sun PZ, Cheung JS, Wang E, et al. Fast multislice pH-weighted chemical exchange saturation transfer (CEST) MRI with unevenly segmented RF irradiation. Magn Reson Med. 2011; 65(2):588-94.

74. Jones CK, Polders D, Hua J, et al. In vivo three-dimensional whole-brain pulsed steady-state chemical exchange saturation transfer at 7T. Magn Reson Med. 2012;67(6):1579-89.

75. Jin T, Wang P, Zong X, Kim S-G. MR imaging of the amideproton transfer effect and the $\mathrm{pH}$-insensitive nuclear overhauser effect at 9.4T. Magn Reson Med. 2012;. doi:10.1002/mrm.24315. 
76. Zaiss M, Schmitt B, Bachert P. Quantitative separation of CEST effect from magnetization transfer and spillover effects by Lorentzian-line-fit analysis of z-spectra. J Magn Reson. 2011;211(2):149-55.

77. Yadav NN, Jones CK, Xu J, et al. Detection of rapidly exchanging compounds using on-resonance frequency-labeled exchange (FLEX) transfer. Magn Reson Med. 2012;68(4):1048-55.

78. Kogan F, Singh A, Cai K, et al. Investigation of chemical exchange at intermediate exchange rates using a combination of chemical exchange saturation transfer (CEST) and spin-locking methods (CESTrho). Magn Reson Med. 2012;68(1):107-19.
79. Song X, Gilad AA, Joel S, et al. CEST phase mapping using a length and offset varied saturation (LOVARS) scheme. Magn Reson Med. 2012;68(4):1074-86.

80. Lee J-S, Regatte RR, Jerschow A. Isolating chemical exchange saturation transfer contrast from magnetization transfer asymmetry under two-frequency $\mathrm{rf}$ irradiation. J Magn Reson. 2012;215:56-63.

81. $\mathrm{Zu} \mathrm{Z}$, Janve VA, Xu J, et al. A new method for detecting exchanging amide protons using chemical exchange rotation transfer. Magn Reson Med. 2012;. doi:10.1002/mrm.24284. 